Proyecciones Journal of Mathematics

Vol. 36, N ${ }^{o}$ 2, pp. 347-361, June 2017.

Universidad Católica del Norte

Antofagasta - Chile

\title{
Skolem difference mean labeling of disconnected graphs
}

\author{
P. Jeyanthi \\ Govindammal Aditanar College for Women, India \\ M. Selvi \\ Dr. Sivanthi Aditanar College of Engineering, India and \\ D. Ramya \\ Govenrnment Arts College for Women, India \\ Received: January 2017. Accepted: March 2017
}

\begin{abstract}
Let $G=(V, E)$ be a graph with $p$ vertices and $q$ edges. $G$ is said to have skolem difference mean labeling if it is possible to label the vertices $x \in V$ with distinct elements $f(x)$ from $1,2,3, \ldots, p+q$ in such a way that for each edge $e=u v$, let $f^{*}(e)=\left\lceil\frac{|f(u)-f(v)|}{2}\right\rceil$ and the resulting labels of the edges are distinct and are from $1,2,3, \ldots, q$. A graph that admits a skolem difference mean labeling is called a skolem difference mean graph. In this paper, we prove that the graphs $C_{m} \cup$ $C_{n}(n, m \geq 3$ and $m \leq n), F_{n} \cup(n-2) K_{2}(n>2),\left(P_{n}+\overline{K_{2}}\right) \cup(2 n-$ $3) K_{2}(n \geq 2)$ and $W_{n} \cup(n-1) K_{2}(n \geq 3)$ are skolem difference mean graphs.
\end{abstract}

Keywords: mean labeling, skolem difference mean labeling, skolem difference mean graph.

AMS Subject Classification: $05 C 78$ 


\section{Introduction}

Throughout this paper by a graph we mean a finite, simple and undirected one. The vertex set and the edge set of a graph $G$ are denoted by $V(G)$ and $E(G)$ respectively. A graph labeling is an assignment of integers to the vertices or edges or both, subject to certain conditions. Terms and notations not defined here are used in the sense of Harary[1]. There are several types of labeling. An excellent survey of graph labeling is maintained by Gallian[2]. The notion of mean labeling was due to Somasundaram et al.[8]. A graph $G=(V, E)$ with $p$ vertices and $q$ edges is called a mean graph if there is an injective function $f$ that maps $V(G)$ to $\{0,1,2, \ldots, q\}$ such that for each edge $u v$, labeled with $\frac{f(u)+f(v)}{2}$ if $f(u)+f(v)$ is even and $\frac{f(u)+f(v)+1}{2}$ if $f(u)+f(v)$ is odd. Then the resulting edge labels are distinct. The concept of skolem difference mean labeling was introduced by Murugan et al.[4] and they studied the skolem difference mean labeling of H-graphs. In[3], they studied skolem difference mean labeling of finite union of paths. Further, the skolem difference mean labeling of $K_{n}(n \geq 3), K_{m, n}(m, n \geq 2), G \cup \overline{K_{n}},\left(G_{1}\right)_{f} *\left(G_{2}\right)_{g}$, $G \cup H$ were proved in[5]. Ramya et al. [6], proved that $\left\langle T \widehat{o} K_{1, n}\right\rangle$, where $\mathrm{T}$ is a $T p$-tree, caterpillar, $S_{m, n}$ and $C_{n} @ K_{1, m}$ were skolem difference mean graphs. In [7], we proved that the graphs $C_{n} @ P_{m}(n \geq 3, m \geq 1)$, $T\left\langle K_{1, n_{1}}: K_{1, n_{2}}: \ldots: K_{1, n_{m}}\right\rangle, T\left\langle K_{1, n_{1}} \circ K_{1, n_{2}} \circ \circ \circ K_{1, n_{m}}\right\rangle, \operatorname{st}\left(n_{1}, n_{2}, \ldots, n_{m}\right)$ and $B t(n, n, \ldots n)$ admit skolem difference mean labeling.

We use the following definitions in the subsequent section.

Definition 1.1. A graph $G=(V, E)$ with $p$ vertices and $q$ edges is said to have skolem difference mean labeling if it is possible to label the vertices $x \in V$ with distinct elements $f(x)$ from $1,2,3, \ldots, p+q$ in such a way that for each edge $e=u v$, let $f^{*}(e)=\left\lceil\frac{|f(u)-f(v)|}{2}\right\rceil$ if $|f(u)-f(v)|$ is even and $\frac{|f(u)-f(v)|+1}{2}$ if $|f(u)-f(v)|$ is odd and the resulting labels of the edges are distinct and are from $1,2,3, \ldots, q$. A graph that admits a skolem difference mean labeling is called a skolem difference mean graph.

Definition 1.2. Let $G_{1}$ and $G_{2}$ be two graphs having vertex sets $V_{1}$ and $V_{2}$ and edge sets $E_{1}$ and $E_{2}$ respectively. Then their union $G_{1} \cup G_{2}$ has $V=V_{1} \cup V_{2}$ and $E=E_{1} \cup E_{2}$.

Definition 1.3. A path is a walk if all the vertices and edges are distinct. A path on $n$ vertices is denoted by $P_{n}$. The graph $m P_{n}$ is the disjoint union of $m$ copies of the path $P_{n}$. 
Definition 1.4. The wheel graph $W_{n}$ is obtained by joining a central vertex to all vertices of the cycle $C_{n}$ by an edge.

Definition 1.5. The fan graph $F_{n}$ is obtained by joining a vertex to all vertices of the path $P_{n}$ by an edge.

\section{Main results}

In this section we prove that $C_{m} \cup C_{n}(n, m \geq 3$ and $m \leq n), F_{n} \cup(n-$ $2) K_{2}(n>2),\left(P_{n}+\overline{K_{2}}\right) \cup(2 n-3) K_{2}(n \geq 2)$ and $W_{n} \cup(n-1) K_{2}(n \geq 3)$ are skolem difference mean graphs.

Theorem 2.1. The union of two cycles $C_{m} \cup C_{n}(n, m \geq 3$ and $m \leq n)$ is a skolem difference mean graph.

Proof. Case(i). $m$ is odd.

Let $m=2 l+1$.

Subcase(i). $n$ is odd.

Let $n=2 k+1$

Let $u_{1}, u_{2}, . ., u_{l}, v_{l}, v_{l-1}, \ldots, v_{1}, v_{0}$ be the vetices of $C_{2 l+1}$ and $u_{1}^{\prime}, u_{2}^{\prime}, \ldots, u_{k}^{\prime}, v_{k}^{\prime}$, $v_{k-1}^{\prime}, \ldots, v_{1}^{\prime}, v_{0}^{\prime}$ be the vertices of $C_{2 k+1}$ respectively.

Then $E\left(C_{m} \cup C_{n}\right)=\left\{v_{i} v_{i+1}: 1 \leq i \leq l-1\right\} \cup\left\{u_{i} u_{i+1}: 1 \leq\right.$ $i \leq l-1\} \cup\left\{v_{i}^{\prime} v_{i+1}^{\prime}: 1 \leq i \leq k-1\right\} \cup\left\{u_{i}^{\prime} u_{i+1}^{\prime}: 1 \leq i \leq k-1\right\} \cup$ $\left\{v_{0} v_{1}, v_{0} u_{1}, u_{l} v_{l}, v_{0}^{\prime} v_{1}^{\prime}, v_{0}^{\prime} u_{1}^{\prime}, u_{k}^{\prime} v_{k}^{\prime}\right\}$. Define $f: V\left(C_{m} \cup C_{n}\right) \rightarrow\{1,2,3, \ldots, p+$ $q=2(m+n)\}$ as follows:

$$
\begin{aligned}
& f\left(v_{0}\right)=1, \\
& f\left(v_{i}\right)= \begin{cases}2(m+n)-2 i+1 & \text { if } i \text { is odd and } 1 \leq i \leq l \\
2 i+1 & \text { if } i \text { is even and } 2 \leq i \leq l,\end{cases} \\
& f\left(u_{i}\right)= \begin{cases}2(m+n)-2 i+2 & \text { if } i \text { is odd and } 1 \leq i \leq l \\
2 i & \text { if } i \text { is even and } 2 \leq i \leq l,\end{cases}
\end{aligned}
$$

For $n=3, f\left(v_{0}^{\prime}\right)=2, f\left(v_{1}^{\prime}\right)=7, f\left(u_{1}^{\prime}\right)=10$.

When $n>3, f\left(v_{0}^{\prime}\right)=3$, 


$$
\begin{aligned}
& f\left(v_{i}^{\prime}\right)= \begin{cases}2 n-2 i+5 & \text { if } i \text { is odd and } 1 \leq i \leq k-1 \\
2 i+3 & \text { if } i \text { is even and } 2 \leq i \leq k-1,\end{cases} \\
& f\left(u_{i}^{\prime}\right)= \begin{cases}2 n-2 i+6 & \text { if } \quad i \text { is odd and } 1 \leq i \leq k-1 \\
2 i+2 & \text { if } \quad i \text { is even and } 2 \leq i \leq k-1,\end{cases} \\
& f\left(v_{k}^{\prime}\right)=\left\{\begin{array}{lll}
n+7 & \text { if } & k \text { is odd } \\
n+1 & \text { if } & k \text { is even, }
\end{array}\right. \\
& f\left(u_{k}^{\prime}\right)=n+4 .
\end{aligned}
$$

For each vertex label $f$, the induced edge label $f^{*}$ is calculated as follows:

$$
\begin{array}{lr}
f^{*}\left(v_{i} v_{i+1}\right)=m+n-2 i-1 & \text { for } 1 \leq i \leq l-1, \\
f^{*}\left(u_{i} u_{i+1}\right)=m+n-2 i & \text { for } 1 \leq i \leq l-1, \\
f^{*}\left(v_{0} v_{1}\right)=n+m-1, f^{*}\left(u_{1} v_{0}\right)=n+m, f^{*}\left(u_{l} v_{l}\right)=1, \\
f^{*}\left(v_{j}^{\prime} v_{j+1}^{\prime}\right)=n-2 j & \text { for } 1 \leq j \leq k-2, \\
f^{*}\left(u_{j}^{\prime} u_{j+1}^{\prime}\right)=n-2 j+1 & \text { for } 1 \leq j \leq k-2, \\
f^{*}\left(v_{0}^{\prime} v_{1}^{\prime}\right)=n, f^{*}\left(v_{0}^{\prime} u_{1}^{\prime}\right)=n+1, f^{*}\left(v_{k}^{\prime} u_{k}^{\prime}\right)=2, \\
f^{*}\left(u_{k-1}^{\prime} u_{k}^{\prime}\right)=3 \text { and } f^{*}\left(v_{k-1}^{\prime} v_{k}^{\prime}\right)=4 . &
\end{array}
$$

Therefore, $E\left(C_{m} \cup C_{n}\right)=\{1,2,3, \ldots, q\}$ and hence $f$ is a skolem difference mean labeling.

Subcase(ii). $n$ is even.

Let $n=2 k, k>1$.

Let $u_{1}, u_{2}, . ., u_{l}, v_{l}, v_{l-1}, \ldots, v_{1}, v_{0}$ be the vetices of $C_{2 l+1}$ and $u_{1}^{\prime}, u_{2}^{\prime}, \ldots, u_{k-2}^{\prime}, u_{k-1}^{\prime}, u_{0}^{\prime}, v_{k-1}^{\prime} \ldots, v_{1}^{\prime}, v_{0}^{\prime}$ be the vertices of $C_{2 k}$ respectively.

Then $E\left(C_{m} \cup C_{n}\right)=\left\{v_{i} v_{i+1}: 1 \leq i \leq l-1\right\} \cup\left\{u_{i} u_{i+1}: 1 \leq i \leq\right.$ $l-1\} \cup\left\{v_{j}^{\prime} v_{j+1}^{\prime}: 1 \leq j \leq k-2\right\} \cup\left\{u_{j}^{\prime} u_{j+1}^{\prime}: 1 \leq j \leq k-2\right\} \cup$ $\left\{v_{0} v_{1}, v_{0} u_{1}, u_{l} v_{l}, v_{0}^{\prime} v_{1}^{\prime}, v_{0}^{\prime} u_{1}^{\prime}, u_{0}^{\prime} u_{k-1}^{\prime}, u_{0}^{\prime} v_{k-1}^{\prime}\right\}$.

Define $f: V\left(C_{m} \cup C_{n}\right) \rightarrow\{1,2,3, \ldots, p+q=2(m+n)\}$ as follows:

$$
f\left(v_{0}\right)=1,
$$




$$
\begin{aligned}
& f\left(v_{i}\right)= \begin{cases}2(m+n)-2 i+1 & \text { if } \quad i \text { is odd and } 1 \leq i \leq l \\
2 i+1 & \text { if } \quad i \text { is even and } 2 \leq i \leq l,\end{cases} \\
& f\left(u_{i}\right)= \begin{cases}2(m+n)-2 i+2 & \text { if } \quad i \text { is odd and } 1 \leq i \leq l \\
2 i & \text { if } \quad i \text { is even and } 2 \leq i \leq l,\end{cases} \\
& f\left(v_{0}^{\prime}\right)=3 \text {, } \\
& f\left(u_{0}^{\prime}\right)=\left\{\begin{array}{lll}
n+5 & \text { if } \quad k \text { is odd } \\
n+3 & \text { if } k \text { is even },
\end{array}\right. \\
& f\left(v_{j}^{\prime}\right)= \begin{cases}2 n-2 j+5 & \text { if } \quad j \text { is odd and } 1 \leq j \leq k-1 \\
2 j+3 & \text { if } \quad j \text { is even and } 2 \leq j \leq k-1,\end{cases} \\
& f\left(u_{j}^{\prime}\right)= \begin{cases}2 n-2 j+6 & \text { if } \quad j \text { is odd and } 1 \leq j \leq k-1 \\
2 j+2 & \text { if } j \text { is even and } 2 \leq j \leq k-1,\end{cases}
\end{aligned}
$$

For each vertex label $f$, the induced edge label $f^{*}$ is calculated as follows:

$f^{*}\left(v_{i} v_{i+1}\right)=m+n-2 i-1 \quad$ for $1 \leq i \leq l-1$, $f^{*}\left(u_{i} u_{i+1}\right)=m+n-2 i \quad$ for $1 \leq i \leq l-1$, $f^{*}\left(v_{0} v_{1}\right)=n+m-1, f^{*}\left(u_{1} v_{0}\right)=n+m, f^{*}\left(u_{l} v_{l}\right)=1$, $f^{*}\left(v_{j}^{\prime} v_{j+1}^{\prime}\right)=n-2 j \quad$ for $1 \leq j \leq k-2$, $f^{*}\left(u_{j}^{\prime} u_{j+1}^{\prime}\right)=n-2 j+1 \quad$ for $1 \leq j \leq k-2$, $f^{*}\left(u_{0}^{\prime} v_{k-1}^{\prime}\right)=2, f^{*}\left(u_{0}^{\prime} u_{k-1}^{\prime}\right)=3, f^{*}\left(v_{0}^{\prime} v_{1}^{\prime}\right)=n$ and $f^{*}\left(v_{0}^{\prime} u_{1}^{\prime}\right)=n+1$.

Therefore, we get the edge labels are from $\{1,2,3, \ldots, q\}$. Hence $f$ is a skolem difference mean labeling.

Case(ii). $m$ is even.

Let $m=2 l$.

Subcase(i). $n$ is odd. 
Let $n=2 k+1$.

Let $u_{1}, u_{2}, . ., u_{l-1}, u_{0}, v_{l-1}, \ldots, v_{1}, v_{0}$ be the vertices of $C_{2 l}$ and $u_{1}^{\prime}, u_{2}^{\prime}, \ldots, u_{k}^{\prime}, v_{k}^{\prime}, v_{k-1}^{\prime}, \ldots, v_{1}^{\prime}, v_{0}^{\prime}$ be the vertices of $C_{2 k+1}$ respectively.

Then $E\left(C_{m} \cup C_{n}\right)=\left\{v_{i} v_{i+1}: 1 \leq i \leq l-2\right\} \cup\left\{u_{i} u_{i+1}: 1 \leq i \leq\right.$ $l-2\} \cup\left\{v_{j}^{\prime} v_{j+1}^{\prime}: 1 \leq j \leq k-1\right\} \cup\left\{u_{j}^{\prime} u_{j+1}^{\prime}: 1 \leq j \leq k-1\right\} \cup$ $\left\{v_{0} v_{1}, v_{0} u_{1}, u_{l-1} u_{0}, u_{0}^{\prime} v_{l-1}^{\prime}, v_{0}^{\prime} v_{1}^{\prime}, v_{0}^{\prime} u_{1}^{\prime}, u_{k}^{\prime} v_{k}^{\prime}\right\}$.

Define $f: V\left(C_{m} \cup C_{n}\right) \rightarrow\{1,2,3, \ldots, p+q=2(m+n)\}$ as follows:

$$
\begin{aligned}
& f\left(v_{0}\right)=1, \\
& f\left(v_{i}\right)= \begin{cases}2(m+n)-2 i+1 & \text { if } \quad i \text { is odd and } 1 \leq i \leq l-1 \\
2 i+1 & \text { if } \quad i \text { is even and } 2 \leq i \leq l-1,\end{cases} \\
& f\left(u_{i}\right)= \begin{cases}2(m+n)-2 i+2 & \text { if } \quad i \text { is odd and } 1 \leq i \leq l-1 \\
2 i & \text { if } \quad i \text { is even and } 2 \leq i \leq l-1,\end{cases} \\
& f\left(u_{0}\right)= \begin{cases}m+2 n+1 & \text { if } \quad l \text { is odd } \\
m+1 & \text { if } \quad l \text { is even },\end{cases} \\
& f\left(v_{0}^{\prime}\right)=3, \quad \text { if } j \text { is odd and } 1 \leq j \leq k \\
& f\left(v_{j}^{\prime}\right)= \begin{cases}2 n-2 j+3 & j \text { is even and } 2 \leq j \leq k, \\
2 j+3 & \text { if } \quad j \text { is odd and } 1 \leq j \leq k\end{cases} \\
& f\left(u_{j}^{\prime}\right)= \begin{cases}2 n-2 j+4 \\
2 j+2 & \text { if } j \text { is even and } 2 \leq j \leq k .\end{cases}
\end{aligned}
$$

For each vertex label $f$, the induced edge label $f^{*}$ is calculated as follows:

$$
\begin{array}{lr}
f^{*}\left(v_{i} v_{i+1}\right)=m+n-2 i-1 & \text { for } 1 \leq i \leq l-2, \\
f^{*}\left(u_{i} u_{i+1}\right)=m+n-2 i & \text { for } 1 \leq i \leq l-2, \\
f^{*}\left(v_{0} v_{1}\right)=n+m-1, f^{*}\left(u_{1} v_{0}\right)=n+m, & \\
f^{*}\left(v_{l-1} u_{0}\right)=n+1, f^{*}\left(u_{0} u_{l-1}\right)=n+2, & \text { for } 1 \leq j \leq k-1, \\
f^{*}\left(v_{j}^{\prime} v_{j+1}^{\prime}\right)=n-2 j-1 & \text { for } 1 \leq j \leq k-1, \\
f^{*}\left(u_{j}^{\prime} u_{j+1}^{\prime}\right)=n-2 j &
\end{array}
$$


$f^{*}\left(v_{0}^{\prime} v_{1}^{\prime}\right)=n-1, f^{*}\left(v_{0}^{\prime} u_{1}^{\prime}\right)=n, f^{*}\left(v_{k}^{\prime} u_{k}^{\prime}\right)=1$.

Therefore, $f$ is a skolem difference mean labeling.

Subcase(ii). $n$ is even.

Let $n=2 k, k>1$.

Let $u_{1}, u_{2}, . ., u_{l-1}, u_{o}, v_{l-1}, \ldots, v_{1}, v_{0}$ be the vertices of $C_{2 l}$ and $u_{1}^{\prime}, u_{2}^{\prime}, \ldots, u_{k-2}^{\prime}$, $u_{k-1}^{\prime}, u_{0}^{\prime}, v_{k-1}^{\prime} \ldots, v_{1}^{\prime}, v_{0}^{\prime}$ be the vertices of $C_{2 k}$ respectively.

Then $E\left(C_{m} \cup C_{n}\right)=\left\{v_{i} v_{i+1}: 1 \leq i \leq l-2\right\} \cup\left\{u_{i} u_{i+1}: 1 \leq i \leq\right.$ $l-2\} \cup\left\{v_{j}^{\prime} v_{j+1}^{\prime}: 1 \leq j \leq k-2\right\} \cup\left\{u_{j}^{\prime} u_{j+1}^{\prime}: 1 \leq j \leq k-2\right\} \cup$ $\left\{v_{0} v_{1}, v_{0} u_{1}, u_{0} v_{l-1}, u_{0} u_{l-1}, v_{0}^{\prime} v_{1}^{\prime}, v_{0}^{\prime} u_{1}^{\prime}, u_{0}^{\prime} u_{k-1}^{\prime}, u_{0}^{\prime} v_{k-1}^{\prime}\right\}$.

Define $f: V\left(C_{m} \cup C_{n}\right) \rightarrow\{1,2,3, \ldots, p+q=2(m+n)\}$ as follows:

$$
\begin{aligned}
& f\left(v_{0}\right)=1 \text {, } \\
& f\left(v_{i}\right)= \begin{cases}2(m+n)-2 i+1 & \text { if } \quad i \text { is odd and } 1 \leq i \leq l-1 \\
2 i+1 & \text { if } \quad i \text { is even and } 2 \leq i \leq l-1,\end{cases} \\
& f\left(u_{i}\right)= \begin{cases}2(m+n)-2 i+2 & \text { if } \quad \mathrm{i} \text { is odd and } 1 \leq i \leq l-1 \\
2 i & \text { if } \quad \mathrm{i} \text { is even and } 1 \leq i \leq l-1,\end{cases} \\
& f\left(u_{0}\right)= \begin{cases}m+2 n+1 & \text { if } \quad l \text { is odd } \\
m+1 & \text { if } l \text { is even , }\end{cases} \\
& f\left(v_{0}^{\prime}\right)=3 \text {, } \\
& f\left(u_{0}^{\prime}\right)=n+3 \\
& f\left(v_{j}^{\prime}\right)=\left\{\begin{array}{lll}
2 n-2 j+3 & \text { if } \quad j \text { is odd and } 1 \leq j \leq k-1 \\
2 j+3 & \text { if } \quad j \text { is even and } 1 \leq j \leq k-1,
\end{array}\right. \\
& f\left(v_{k-1}^{\prime}\right)= \begin{cases}n+2 & \text { if } \quad \mathrm{k} \text { is odd } \\
n+4 & \text { if } \mathrm{k} \text { is even }\end{cases}
\end{aligned}
$$




$$
f\left(u_{j}^{\prime}\right)= \begin{cases}2 n-2 j+4 & \text { if } \quad j \text { is odd and } 1 \leq j \leq k-1 \\ 2 j+2 & \text { if } \quad j \text { is even and } 2 \leq j \leq k-1 .\end{cases}
$$

For each vertex label $f$, the induced edge label $f^{*}$ is calculated as follows:

$f^{*}\left(v_{i} v_{i+1}\right)=m+n-2 i-1 \quad$ for $1 \leq i \leq l-2$,

$f^{*}\left(u_{i} u_{i+1}\right)=m+n-2 i \quad$ for $1 \leq i \leq l-2$,

$f^{*}\left(v_{0} v_{1}\right)=n+m-1, f^{*}\left(u_{1} v_{0}\right)=n+m, f^{*}\left(u_{0} v_{l-1}\right)=n+1, f^{*}\left(u_{0} u_{l-1}\right)=$ $n+2$,

$f^{*}\left(v_{j}^{\prime} v_{j+1}^{\prime}\right)=n-2 j-1 \quad$ for $1 \leq j \leq k-2$,

$f^{*}\left(u_{j}^{\prime} u_{j+1}^{\prime}\right)=n-2 j \quad$ for $1 \leq j \leq k-2$,

$f^{*}\left(v_{0}^{\prime} v_{1}^{\prime}\right)=n-1, f^{*}\left(v_{0}^{\prime} u_{1}^{\prime}\right)=n, f^{*}\left(v_{k-1}^{\prime} u_{0}^{\prime}\right)=1, f^{*}\left(u_{0}^{\prime} u_{k-1}^{\prime}\right)=2$.

Therefore, $f$ is a skolem difference mean labeling and hence $C_{m} \cup C_{n}$ is a skolem difference mean graph. The skolem difference mean labeling of $C_{7} \cup C_{8}$ and $C_{9} \cup C_{3}$ are shown in Figure 1 and 2 .
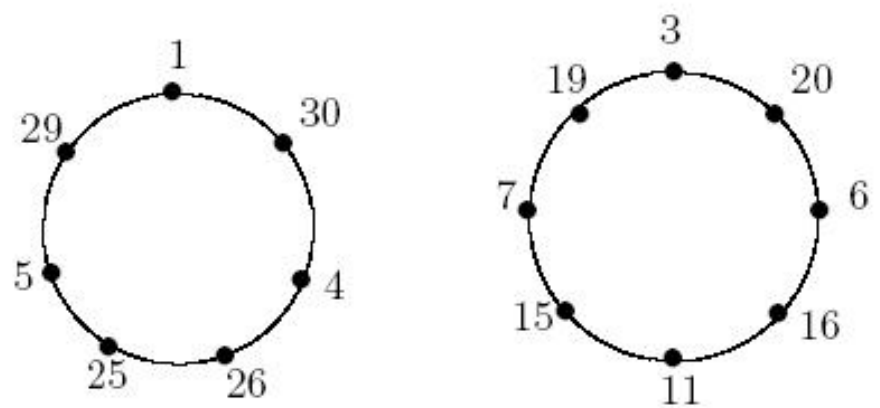

Figure 1: $C_{7} \cup C_{8}$
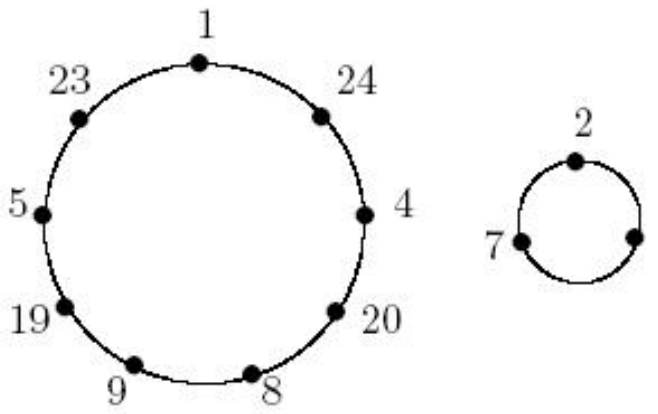

Figure 2: $C_{9} \cup C_{3}$ 
Theorem 2.2. The graph $F_{n} \cup(n-2) K_{2},(n>2)$ is a skolem difference mean graph.

Proof. Let $v_{0}, v_{i}(1 \leq i \leq n)$ be the vertices of $F_{n}$ and $x_{j}, y_{j}(1 \leq j \leq$ $n-2)$ be the vertices of $(n-2) K_{2}$ respectively.

Then $E\left(F_{n} \cup(n-2) K_{2}\right)=\left\{v_{i} v_{i+1}: 1 \leq i \leq n-1\right\} \cup\left\{v_{0} v_{i}: 1 \leq i \leq\right.$ $n\} \cup\left\{x_{j} y_{j}: 1 \leq j \leq(n-2)\right\}$.

Define $f: V\left(F_{n} \cup(n-2) K_{2}\right) \rightarrow\{1,2,3, \ldots, p+q=6(n-1)\}$ as follows:

$f\left(v_{0}\right)=5 n-4$

$f\left(v_{i}\right)=n-2+i \quad$ if $i$ is odd and $1 \leq i \leq n$, $f\left(v_{i}\right)=3 n-2-i \quad$ if $i$ is even and $2 \leq i \leq n$, $f\left(x_{j}\right)=j \quad$ for $1 \leq j \leq n-2$,

$f\left(y_{j}\right)=6 n-5-j \quad$ for $1 \leq j \leq n-2$.

Let $e_{i}=v_{0} v_{i}, e_{i}^{\prime}=v_{i} v_{i+1}(1 \leq i \leq n-1)$ and $e_{j}=x_{j} y_{j}(1 \leq j \leq n-2)$.

For each vertex label $f$, the induced edge label $f^{*}$ is calculated as follows:

$f^{*}\left(e_{j}\right)=3 n-2-j$

for $1 \leq j \leq n-2$,

$f^{*}\left(e_{i}^{\prime}\right)=n-i$

$f^{*}\left(e_{i}\right)=2 n-\left(\frac{i+1}{2}\right)$

for $1 \leq i \leq n-1$,

$f^{*}\left(e_{i}\right)=n+\left(\frac{i-2}{2}\right)$

if $i$ is odd and $1 \leq i \leq n$,

if $i$ is even and $2 \leq i \leq n$.

Therefore, $f$ is a skolem difference mean labeling of $F_{n} \cup(n-2) K_{2}$ and hence $F_{n} \cup(n-2) K_{2}(n>2)$ is a skolem difference mean graph.

A skolem difference mean labeling of $F_{7} \cup 5 K_{2}$ is shown in Figure 3 . 

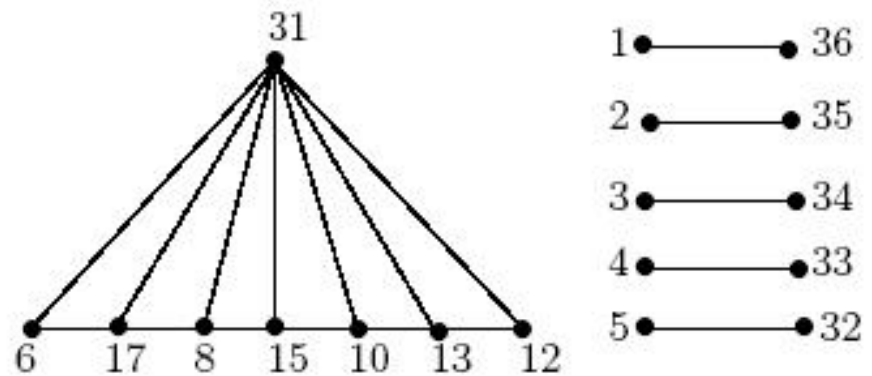

Figure 3: $F_{7} \cup 5 K_{2}$

Theorem 2.3. The graph $\left(P_{n}+\overline{K_{2}}\right) \cup(2 n-3) K_{2},(n \geq 2)$ is a skolem difference mean graph.

Proof. Let $u_{0}, v_{0}, v_{i}(1 \leq i \leq n)$ be the vertices of $\left(P_{n}+\overline{K_{2}}\right)$ and $x_{t}, y_{t}$ $(1 \leq t \leq 2 n-3)$ be the vertices of $(2 n-3) K_{2}$ respectively.

Then $E\left(\left(P_{n}+\overline{K_{2}}\right) \cup(2 n-3) K_{2}\right)=\left\{v_{i} v_{i+1}: 1 \leq i \leq n-1\right\} \cup\left\{u_{0} v_{i}, v_{0} v_{i}\right.$ : $1 \leq i \leq n\} \cup\left\{x_{t} y_{t}: 1 \leq t \leq(2 n-3)\right\}$.

Define $f: V\left(\left(P_{n}+\overline{K_{2}}\right) \cup(2 n-3) K_{2}\right) \rightarrow\{1,2,3, \ldots, p+q=10 n-8\}$ as follows:

$$
f\left(v_{0}\right)=8 n-5, f\left(u_{0}\right)=6 n-5,
$$

$f\left(v_{i}\right)=2 n-3+i \quad$ if $i$ is odd and $1 \leq i \leq n$,

$f\left(v_{i}\right)=4 n-2-i \quad$ if $i$ is even and $2 \leq i \leq n$,

$f\left(x_{t}\right)=t \quad$ for $1 \leq t \leq 2 n-3$,

$f\left(y_{t}\right)=10 n-7-t \quad$ for $1 \leq t \leq 2 n-3$.

Let $e_{i}=v_{0} v_{i}, e_{i}^{\prime}=u_{0} v_{i}(1 \leq i \leq n), e_{j}=v_{j} v_{j+1}(1 \leq j \leq n-1)$ and $e_{t}=x_{t} y_{t}(1 \leq t \leq 2 n-3)$.

For each vertex label $f$, the induced edge label $f^{*}$ is calculated as follows:

$f^{*}\left(e_{t}\right)=5 n-3-t$

for $1 \leq t \leq 2 n-3$,

$f^{*}\left(e_{j}\right)=n-j$

for $1 \leq j \leq n-1$, 
$f^{*}\left(e_{i}\right)=3 n-\left(\frac{i+1}{2}\right)$

$f^{*}\left(e_{i}\right)=2 n+\left(\frac{i-2}{2}\right)$

$f^{*}\left(e_{i}^{\prime}\right)=2 n-\left(\frac{i+1}{2}\right)$

$f^{*}\left(e_{i}^{\prime}\right)=n+\left(\frac{i-2}{2}\right)$ if $i$ is odd and $1 \leq i \leq n$,

if $i$ is even and $1 \leq i \leq n$,

if $i$ is odd and $1 \leq i \leq n$,

if $i$ is even and $1 \leq i \leq n$.

Therefore, $f$ is a skolem difference mean labeling of $\left(P_{n}+\overline{K_{2}}\right) \cup(2 n-$ $3) K_{2}$ and hence $\left(P_{n}+\overline{K_{2}}\right) \cup(2 n-3) K_{2}$ is a skolem difference mean graph.

A skolem difference mean labeling of $\left(P_{7}+\overline{K_{2}}\right) \cup 11 K_{2}$ is shown in Figure 4.
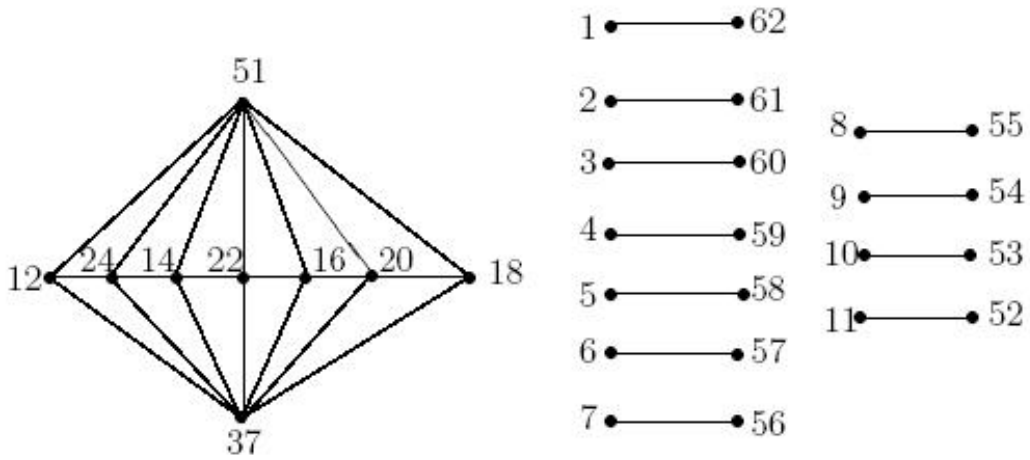

Figure 4: $\left(P_{7}+\overline{K_{2}}\right) \cup 11 K_{2}$

Theorem 2.4. The graph $W_{n} \cup(n-1) K_{2},(n \geq 3)$ is a skolem difference mean graph.

Proof. Let $v_{0}, v_{i}(1 \leq i \leq n)$ be the vertices of $W_{n}$ and $x_{t}, y_{t}(1 \leq t \leq$ $n-1)$ be the vertices of $(n-1) K_{2}$ respectively.

Then $E\left(W_{n} \cup(n-1) K_{2}\right)=\left\{v_{i} v_{i+1}: 1 \leq i \leq n-1\right\} \cup\left\{v_{0} v_{i}: 1 \leq i \leq\right.$ $n\} \cup\left\{x_{t} y_{t}: 1 \leq t \leq(n-1)\right\} \cup\left\{v_{n} v_{1}\right\}$.

Define $f: V\left(W_{n} \cup(n-1) K_{2}\right) \rightarrow\{1,2,3, \ldots, p+q=6 n-2\}$ as follows:

Case(i). $n$ is odd. 
Let $n=2 k+1$.
$f\left(x_{t}\right)=t$
for $1 \leq t \leq n-2$,
$f\left(x_{n-1}\right)=n$,
$f\left(y_{t}\right)=6 n-t-1$
for $1 \leq t \leq n-2$,
$f\left(y_{n-1}\right)=5 n-1, f\left(v_{0}\right)=5 n, f\left(v_{1}\right)=n-1$,
$f\left(v_{i}\right)=3 n-2 i+2$
if $i$ is even and $2 \leq i \leq n-1$,
$f\left(v_{i}\right)=n+2 i-4$
if $i$ is odd and $3 \leq i \leq k+1$,
$f\left(v_{2 k+2-i}\right)=3 n-2 i-1$
if $i$ is odd and $1 \leq i \leq k$.

For each vertex label $f$, the induced edge label $f^{*}$ is calculated as follows:
$f^{*}\left(x_{t} y_{t}\right)=3 n-t$
for $1 \leq t \leq n-2$,
$f^{*}\left(x_{n-1} y_{n-1}\right)=2 n$,
for $1 \leq i \leq k+1$,
$f^{*}\left(v_{i} v_{i+1}\right)=n-2 i+2$
for $k+2 \leq i \leq 2 k$,
$f^{*}\left(v_{2 k+1} v_{1}\right)=n-1$,
$f^{*}\left(v_{0} v_{i}\right)=2 n+2-i$
if $i$ is odd and $1 \leq i \leq n$,
$f^{*}\left(v_{0} v_{i}\right)=n+i-1$
if $i$ is even and $2 \leq i \leq n$.

Therefore, $E\left(W_{n} \cup(n-1) K_{2}\right)=\{1,2,3, \ldots, q\}$ and hence $f$ is a skolem difference mean labeling.

Case(ii). $n$ is even.

Let $n=2 k, k>1$.
$f\left(x_{t}\right)=t$
for $1 \leq t \leq n-2$,
$f\left(x_{n-1}\right)=n$,
$f\left(y_{t}\right)=6 n-t-1 \quad$ for $1 \leq t \leq n-2$,
$f\left(y_{n-1}\right)=5 n-1, f\left(v_{0}\right)=5 n, f\left(v_{1}\right)=n-1$,
$f\left(v_{i}\right)=3 n-2 i+2$
if $i$ is even and $2 \leq i \leq k$,
$f\left(v_{i}\right)=n+2 i-4$
if $i$ is odd and $3 \leq i \leq k$,
$\mathrm{f}\left(\mathrm{v}_{k+1}\right)=\left\{\begin{array}{lll}2 n-1 & \text { if } \quad k \text { is even } \\ 2 n & \text { if } \quad k \text { is odd, }\end{array}\right.$
$f\left(v_{2 k+1-i}\right)=3 n-2 i-1 \quad$ if $i \quad$ is odd and $1 \leq i \leq k-1$,
$f\left(v_{2 k+1-i}\right)=n+2 i \quad$ if $i$ is even and $2 \leq i \leq k-1$. 
For each vertex label $f$, the induced edge label $f^{*}$ is calculated as follows:

$f^{*}\left(x_{t} y_{t}\right)=3 n-t \quad$ for $1 \leq t \leq n-2$,

$f^{*}\left(x_{n-1} y_{n-1}\right)=2 n$,

$f^{*}\left(v_{i} v_{i+1}\right)=n-2 i+2 \quad$ for $1 \leq i \leq k$,

$f^{*}\left(v_{i} v_{i+1}\right)=2 i-n-1 \quad$ for $k+1 \leq i \leq 2 k-1$,

$f^{*}\left(v_{2 k} v_{1}\right)=n-1$,

$f^{*}\left(v_{0} v_{i}\right)= \begin{cases}2 n+2-i & \text { if } \quad i \text { is odd and } 1 \leq i \leq k+1 \\ n-1+i & \text { if } \quad i \text { is even and } 2 \leq i \leq k+1\end{cases}$

$f^{*}\left(v_{0} v_{2 k+1-i}\right)= \begin{cases}n+1+i & \text { if } \quad i \text { is odd and } 1 \leq i \leq k-1 \\ 2 n-i & \text { if } \quad i \text { is even and } 2 \leq i \leq k-1 .\end{cases}$

Therefore, $f$ is a skolem difference mean labeling and hence $W_{n} \cup(n-1) K_{2}$ is a skolem difference mean graph.

A skolem difference mean labeling of $W_{8} \cup 7 K_{2}$ is shown in Figure 5 .
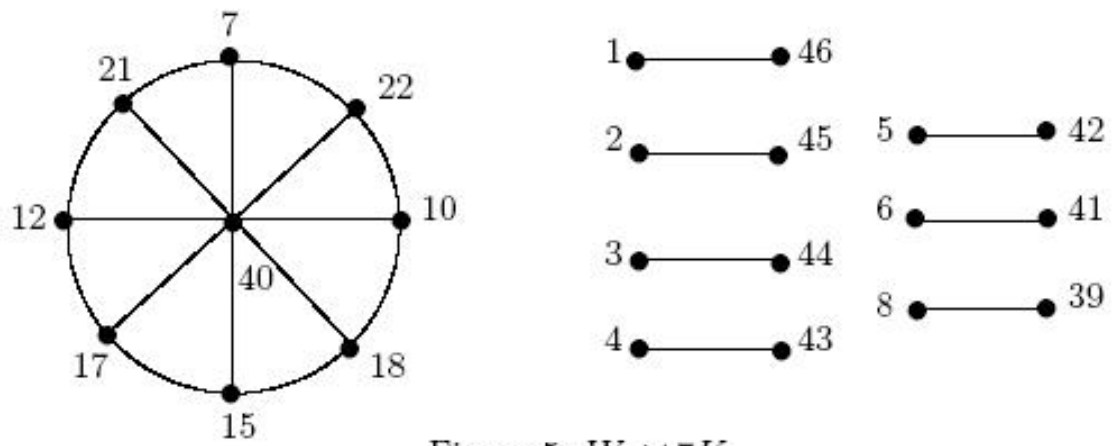

Figure 5: $W_{8} \cup 7 K_{2}$

\section{References}

[1] F. Harary, Graph theory, Addison Wesley, Massachusetts, (1972).

[2] Joseph A. Gallian, A Dynamic Survey of Graph Labeling, The Electronic Journal of Combinatorics, (2015), \#DS6. 
[3] K. Murugan, A. Subramanaian, Labeling of finite union of paths, International Journal of Mathematics and Soft Computing, 2, (1), pp. 99-108, (2012).

[4] K. Murugan, A. Subramanaian, Skolem difference mean labeling of Hgraphs, International Journal of Mathematics and Soft Computing, 1, (1), pp. 115-129, (2011).

[5] K. Murugan, Some Results on Skolem Difference Mean Graphs, International Journal of Mathematics and its Applications, 3, (3-D), pp. 75-80, (2015).

[6] D. Ramya, M. Selvi and R. Kalaiyarasi, On skolem difference mean labeling of graphs, International Journal of Mathematical Archive, 4, (12), pp. 73-79, (2013).

[7] M. Selvi, D. Ramya and P.Jeyanthi, Skolem difference mean Graphs, Proyecciones Journal of Mathematics, 34 (3), pp. 243-254, (2015).

[8] S. Somasundaram and R. Ponraj, Mean labelings of graphs, National Academy Science Letter, (26), pp. 210-213, (2003).

\section{P. Jeyanthi}

Department of Mathematics, Research Centre, Govindammal Aditanar College for Women, Tiruchendur-628215, Tamilnadu, India

e-mail : jeyajeyanthi@rediffmail.com

\section{Selvi}

Department of Mathematics,

Dr. Sivanthi Aditanar College of Engineering, Tiruchendur-628215, Tamilnadu, India

e-mail : selvm80@yahoo.in

and 


\section{Ramya}

Department of Mathematics,

Government Arts College for Women, Ramanathapuram, Tamilnadu,

India

e-mail : aymar_padma@yahoo.co.in 